\title{
ENSAIOS
}

\section{RESILIÊNCA EM SISTEMAS DE VAREJO URBANO}

\section{RESUMO}

Felipe Gerhard Paula Sousa felipegerhard@yahoo.com.br Doutorando em Administração pela Universidade Estadual do Ceará (UECE) - Fortaleza - CE - $B R$.

\section{Verónica Peñaloza} veronica.penaloza@uece.br Doutora em Economia pela Universidade de São Paulo (USP). Professora da Universidade Estadual do Ceará (UECE) - Fortaleza-CE-BR.

Caio Victor de Paula Sousa caiovictor.rns@gmail.com Graduado em Administração pela Universidade Estadual do Ceará (UECE) - Fortaleza - CE - $B R$.

Brenno Buarque de Lima brenno_buarque@hotmail.com Mestrando em Administração pela Universidade Estadual do Ceará (UECE) - Fortaleza - CE - BR.
Este ensaio tem como objetivo geral investigar as principais propriedades e características relacionadas à resiliência em sistemas de varejo urbano. Para tal, por meio da análise de aspectos teóricos concernentes à resiliência em estudos de diversas disciplinas, busca-se erigir um conceito de resiliência específico de sistemas de varejo urbano e analisar criticamente as diferenças teóricas existentes entre os processos de desenvolvimento dos sistemas econômicos e dos sistemas de varejo urbano. Com supedâneo nas análises realizadas, observa-se que, para tais ambientes, resiliência representa a capacidade de se manter ou atingir um equilíbrio dinâmico em harmonia com a reorganização advinda da adaptação às adversidades enfrentadas. A resiliência seria a engrenagem central que faz girar todo o processo de desenvolvimento diacrônico dos sistemas de varejo urbano. Assim, conclui-se que o enfrentamento e a superação tanto das vulnerabilidades presentes no sistema quanto das adversidades provenientes do ambiente externo são importantes propulsores da resiliência.

Palavras-chave: Resiliência. Sistemas de varejo urbano. Equilíbrio dinâmico. Desenvolvimento.

\section{INTRODUÇÃO}

Com o intuito de se manter vantagem competitiva, a mudança e a adaptação são exigidas dos sistemas atuais como um movimento obrigatório, em resposta às flutuações ambientais (MEDD; MARVIN, 2005; BURNARD; BHAMRA, 2011). Destarte, a resiliência, em vista das adversidades que ocasionam ciclos de desenvolvimento deletérios, é recomendada a todos os agentes presentes no mercado, porquanto se caracteriza como mecanismo capaz de habilitar indivíduos, organizações ou sistemas a se adaptarem a mudanças advindas do ambiente externos (BURNARD; BHAMRA, 2011; OZUDURU; VAROL; ERCOSKUN, 2014; PETRESCU; BHATLI, 2013).

Ao longo das últimas décadas, os trabalhos voltados ao aprofundamento de estudos referentes à resiliência vêm ganhando notoriedade, tanto no meio acadêmico quanto para as políticas públicas (MEDD; MARVIN, 2005; SOUMAGNE et al., 2009; STUMPP, 2013). O conceito de resiliência, originalmente cunhado 
nas ciências naturais, vem sofrendo adaptações teóricas para abranger um escopo de pesquisa cada vez maior. Embora tenha ganhado maiores proporções especialmente nas ciências médicas e comportamentais (SMITH et al., 2008), o conceito pôde transitar nas mais distintas ciências, auxiliando sobremaneira as disciplinas e instituições a enfrentarem os problemas teóricos e práticos que lhes são próprios.

Não obstante seja uma qualidade importante que faculta a indivíduos e sistemas proteção contra a adversidade, não há consenso quanto às definições operacionais existentes, uma vez que a literatura concernente ao tema ainda permanece incerta em relação ao acesso da resiliência em diferentes contextos históricos, sociais e culturais (HERRMAN et al., 2011; BUENO, 2012; LEE et al., 2013).

No que concerne aos sistemas de varejo urbano, i.e., aglomerados de negócios interdependentes que compartilham o mesmo espaço físico, infraestrutura e imagem (MEDD; MARVIN, 2005; SOUMAGNE et al., 2009; KÄRRHOLM; NYLUND; DE LA FUENTE, 2014; ERKIP; KIZILGÜN; AKINCI, 2014), ainda não há consenso quanto à definição ideal de resiliência para análises empíricas (HERRMAN et al., 2011; BUENO, 2012). Contudo, um movimento judicioso a tais sistemas é o de autoanálise contínua, cuja função principal é a ciência das vulnerabilidades e fortalezas que permitem a sua sobrevivência (HOLLING, 2001; BHAMRA; DANI; BURNARD, 2011; FRANCIS; BEKERA, 2014). O exame dos principais pontos fracos e fortes de um sistema de varejo urbano, dessa forma, coadjuvaria na construção da ideia de resiliência.

Pode-se constatar a importância que o conceito vem adquirindo no cenário internacional ao se observar a mudança do título do congresso anual da Association of European Planning Schools, passando a se chamar Planning for Resilient Cities and Regions em 2013, devido à relevância que o tema alcançou ao longo dos últimos anos (STUMPP, 2013). No entanto, ainda é pequena a contribuição dos trabalhos nacionais voltados para o exame da resiliência nas ciências sociais aplicadas. Os estudos nacionais se concentram fortemente na área de recursos humanos, tendo como objeto de análise os indivíduos nas organizações; passando ao largo de questões relacionadas à perspectiva urbana e social.

Destarte, com base nas acepções supracitadas, este trabalho tem como objetivo investigar as principais propriedades e características relacionadas à resiliência em sistemas de varejo urbano. Para tal, primeiramente será erigida uma definição de resiliência voltada especificamente para tais ambientes; por conseguinte, serão analisadas as principais propriedades relacionadas à resiliência em sistemas econômicos e, mais especificamente, em sistemas de varejo urbano; e, por fim, realiza-se uma análise crítica concernente aos processos de desenvolvimento dos sistemas econômicos e sistemas de varejo urbano.

Este artigo está divido em quatro seções além desta introdução. Incialmente, será apresentada uma breve síntese das origens e evolução do conceito de resiliência, desde a sua concepção à apropriação científica do termo por diversas disciplinas. Por conseguinte, serão analisadas as definições de resiliência existentes em várias áreas do conhecimento, bem como elementos e características essenciais relacionados ao conceito em sistemas econômicos e sistemas de varejo urbano. Por fim, apresentam-se as considerações finais do estudo, destacando-se as maiores limitações do trabalho e as sugestões para pesquisas futuras.

\section{ORIGENS DO CONCEITO DE RE- SILIÊNCIA}

A etimologia do conceito de resiliência advém do latim resilientia, que por sua vez advém do verbo resilire, o qual possui o significado estrito de saltar para trás; re (prefixo que indica retrocesso) e salire (pular, saltar) (BRANDÃO; MAHFOUD; GIANORDOLI-NASCIMENTO, 2011). A palavra resilientia, dessa forma, apropriando-se do sentido oriundo do verbo, significaria voltar ao normal (SA- 
BBAG et al., 2010). Somente a partir do século XVIII, contudo, o conceito passou a ser utilizado com maior ímpeto, devido, principalmente, aos avanços da ciência. Resilienza, denominação italiana, designava a capacidade de um material em resistir a choques sem romper-se.

As ciências naturais foram as responsáveis pela popularização do termo resiliência, uma vez que a sua definição inicial se associava, principalmente, a resistência de objetos (YUNES, 2003). Atribuindo a propriedade de resiliente a uma amplitude maior de materiais, como, por exemplo, a corpos elásticos, resiliência passou a significar a capacidade de um corpo físico retornar a seu estado normal de equilíbrio após tensões ou estresses (YUNES, 2003; SABBAG et al., 2010). Tal definição passou a ser utilizada posteriormente também pela Engenharia (SABBAG et al., 2010).

Um dos primeiros cientistas a utilizar sistematicamente o termo resiliência foi Thomas Young, no século XIX (TIMOSHENKO, 1983; YUNES, 2003). Young realizou diversos experimentos para testar a resistência de barras ao serem submetidas a altas tensões. $\mathrm{O}$ cientista também foi um dos precursores no estudo dos estresses suportados por corpos físicos, oriundos de impactos planejados. Young chegou a elaborar métodos para o cálculo desses impactos e a resistência obtida pelos materiais estudados (TIMOSHENKO, 1983).

Cumpre salientar que, embora os conceitos de resiliência, vinculados ao estudo das propriedades de resistência de corpos físicos, possuam características semelhantes nas ciências naturais, tais definições destoam entre si. Entendida originalmente como uma propriedade de retornar ao estado normal inicial, a resiliência também é entendida como a capacidade de um material absorver energia, oriunda de uma externalidade, sem sofrer deformações significativas (YUNES, 2003; SABBAG et al., 2010).

Das ciências naturais, o conceito de resiliência foi adaptado para diversas disciplinas, como a Psicologia, as Ciências Médicas e as Ciências Sociais Aplicadas (YUNES, 2003; SMITH et al., 2008). Na Psicologia, o termo ganha amplitude e passa a se referir à capacidade de indivíduos de se recuperar ou resistir a estresses ou situações adversas (YUNES, 2003). As adversidades, nas áreas de estudo comportamentais, são representadas pelas nuances ou riscos externos ao indivíduo. Dessa forma, em consonância com as definições apresentadas até então pelas Ciências Naturais, os indivíduos resistem, protegem-se e superam a influência de fatores externos. Contudo, é importante que se ressalte que a ideia de resiliência na disciplina passa a ser concebida de forma dinâmica, ou seja, o indivíduo não retroage a um estágio anterior de equilíbrio, mas avança para o alcance, através da experiência e da aprendizagem, de níveis superiores de harmonia (SMITH et al., 2008). Dessa forma, ainda que as vicissitudes dos agentes presentes no ambiente externo se lhe apresentem como ameaça, há uma confluência entre indivíduo e ambiente para que se possam atingir patamares mais elevados de equilíbrio (LEE et al., 2013).

Nas Ciências Sociais Aplicadas, o termo resiliência passou a se reportar não somente a indivíduos, mas a grupos, organizações e sistemas econômicos. Contudo, independente do objeto de análise, a resiliência está associada à ideia de se suportar pressões, crises e adversidades (YUNES, 2003). Embora nos últimos anos as ciências econômicas tenham se apropriado grandemente do conceito para embasar análises de mercados, setores e sistemas econômicos (HERRMAN et al., 2011; STUMPP, 2013), foi especialmente nas ciências médicas e comportamentais que a resiliência ganhou maiores proporções (SMITH et al., 2008).

Nos últimos anos, a resiliência também passou a ser estudada no âmbito organizacional, como um fenômeno que fortalece a empresa em meio às tribulações do ambiente externo, tais como crises, mudanças na conjuntura econômica e no contexto legislativo, alterações no cenário social etc. (HAMEL; VALIKANGAS, 2003; POWLEY, 2009). Um dos segmentos que mais concentram estudos sobre resiliência na área organizacional é o de recursos huma- 
nos, com pesquisas que analisam o impacto e a influência do conceito no ambiente de trabalho (HAMEL; VALIKANGAS, 2003).

Em consonância com as pesquisas internacionais, os estudos nacionais em resiliência também se concentram na área de recursos humanos e, em geral, salientam a relevância do conceito para a experiência de socialização em organizações (CARVALHO et al., 2011; SCHERER; MINELLO, 2017). Os artigos ressaltam a importância do suporte psicológico aos colaboradores, facultado pela resiliência individual, para a defesa de ações negativas, bem como mudanças e eventos estressantes nas organizações (CANGUSSU; SACHUK, 2014; BACCHI; PINHEIRO, 2011).

\section{RESILIÊNCIA APLICADA A SISTE- MAS DE VAREJO URBANO}

A seguir, apresenta-se um breve compêndio de estudos relacionados à resiliência, com obras publicadas em diversos campos do conhecimento. Inicialmente, abordar-se-ão as definições de resiliência existentes em diferentes disciplinas com o intuito de se erigir um conceito específico a sistemas de varejo urbano; logo em seguida, analisar-se-ão as propriedades e as características tanto de sistemas econômicos quanto de sistemas de varejo urbano, analisando-se criticamente suas diferenças teóricas.

\subsection{QUADRO CONCEITUAL DE RESI- LIÊNCIA}

Lee et al. (2013), mediante uma análise da literatura referente ao tema, salientam a existência de dois pontos de vista pelos quais se pode definir resiliência. O primeiro concebe o conceito como um traço fixo e estável, referindo-se à capacidade de administrar e adaptar-se a fontes significantes de estresse e descontinuidade. Os autores aduzem como limitações dessa perspectiva à ausência de explanações mais consistentes quanto às interações do elemento com o seu ambiente, como a família, a comunidade ou o sistema social. Na segunda abordagem, a resiliência é vista como um processo permanente, dinâmico e mutável. Configura-se como uma abordagem mais completa, porquanto salienta a dependência do elemento às interações com os vários fatores que a rodeiam. Simmie e Martin (2010) corroboram essa perspectiva ao salientarem que a resiliência, na economia e nas ciências sociais, rejeita abordagens em que um sistema retoma um equilíbrio inicial, argumentando que ambientes e sistemas estão em perpétua mudança. Para os autores, não há estabilidade estática para qualquer elemento, mas um processo dinâmico de equilíbrio.

Com efeito, destaca-se no Quadro 1, a seguir, um recorte de conceitos de resiliência entre a grande pluralidade de definições existentes na literatura, em ordem das ciências que primeiramente a abordaram.

\begin{tabular}{|l|l|l|l|}
\hline \multicolumn{1}{|c|}{ Autor } & Contexto & \multicolumn{1}{|c|}{ Definição } & \multicolumn{1}{|c|}{ Propriedades } \\
\hline $\begin{array}{l}\text { Hollnagel, } \\
\text { Woods, } \\
\text { Leveson } \\
(2006)\end{array}$ & Engenharia & $\begin{array}{l}\text { A capacidade de sentir, reconhecer, adaptar e absorver } \\
\text { variações, mudanças, alterações, interrupções es econhecer, } \\
\text { surpresas }\end{array}$ & $\begin{array}{l}\text { Rectaptar, absorver } \\
\text { adaper }\end{array}$ \\
\hline $\begin{array}{l}\text { Holling } \\
(1973)\end{array}$ & $\begin{array}{l}\text { Sistemas } \\
\text { Ecológicos }\end{array}$ & $\begin{array}{l}\text { A medida da persistência de sistemas e da capacidade } \\
\text { de absorver mudanças e perturbação e ainda manter as } \\
\text { mesmas relações entre as variáveis de estado }\end{array}$ & $\begin{array}{l}\text { Persistir, absorver, } \\
\text { manter }\end{array}$ \\
\hline $\begin{array}{l}\text { Walker et al. } \\
(2004)\end{array}$ & $\begin{array}{l}\text { Sistemas } \\
\text { Ecológicos }\end{array}$ & $\begin{array}{l}\text { A capacidade de um sistema para absorver uma } \\
\text { perturbação e reorganizar enquanto passa por uma } \\
\text { mudança, mantendo a mesma função, estrutura, } \\
\text { identidade e feedback }\end{array}$ & $\begin{array}{l}\text { Absorver, } \\
\text { reorganizar, manter }\end{array}$ \\
\hline
\end{tabular}




\begin{tabular}{|c|c|c|c|}
\hline $\begin{array}{l}\text { Walker et al. } \\
(2002)\end{array}$ & $\begin{array}{l}\text { Sistemas } \\
\text { Socio- } \\
\text { ecológicos }\end{array}$ & $\begin{array}{l}\text { A capacidade para manter a funcionalidade de um sistema } \\
\text { quando é perturbado ou a capacidade de manter os } \\
\text { elementos necessários para renovar ou reorganizar-se se } \\
\text { uma perturbação altera a estrutura da função do sistema }\end{array}$ & $\begin{array}{l}\text { Manter, renovar, } \\
\text { reorganizar }\end{array}$ \\
\hline $\begin{array}{l}\text { Carpenter et } \\
\text { al. }(2001)\end{array}$ & $\begin{array}{l}\text { Sistemas } \\
\text { Socio- } \\
\text { ecológicos }\end{array}$ & $\begin{array}{l}\text { A magnitude de perturbação que um sistema pode } \\
\text { tolerar antes da transição para um estado diferente que é } \\
\text { controlado por um conjunto diferente de processos }\end{array}$ & Tolerar \\
\hline $\begin{array}{l}\text { Luthans, } \\
\text { Vogelgesang e } \\
\text { Lester (2006) }\end{array}$ & Psicologia & $\begin{array}{l}\text { A capacidade de desenvolvimento para reagir à } \\
\text { adversidade }\end{array}$ & Desenvolver, reagir \\
\hline $\begin{array}{l}\text { Rutter } \\
(2006)\end{array}$ & Psicologia & $\begin{array}{l}\text { Um conceito interativo que se refere à relativa } \\
\text { resistência a riscos ambientais ou superação de estresse } \\
\text { ou adversidade }\end{array}$ & Resistir, superar \\
\hline $\begin{array}{l}\text { Lee et al. } \\
(2013)\end{array}$ & Psicologia & $\begin{array}{l}\text { A resiliência pode ser considerada como um processo } \\
\text { dinâmico, que tanto protege o indivíduo em situações } \\
\text { adversas quanto aumenta os seus resultados terapêticos } \\
\text { contra fatores de risco }\end{array}$ & $\begin{array}{l}\text { Proteger, } \\
\text { incrementar } \\
\text { resultados }\end{array}$ \\
\hline $\begin{array}{l}\text { Herrman et } \\
\text { al. }(2011)\end{array}$ & Psicologia & $\begin{array}{l}\text { Resiliência é entendida como referindo-se à adaptação } \\
\text { positiva, ou à capacidade de manter ou recuperar a } \\
\text { saúde mental, apesar de experimentar adversidade }\end{array}$ & $\begin{array}{l}\text { Adaptar, manter, } \\
\text { recuperar }\end{array}$ \\
\hline $\begin{array}{l}\text { Paton, } \\
\text { Smith e Vio- } \\
\text { lanti }(2000)\end{array}$ & $\begin{array}{l}\text { Gestão de } \\
\text { desastres } \\
\text {-individual }\end{array}$ & $\begin{array}{l}\text { Resiliência descreve um processo ativo de } \\
\text { "autoendireitamento", desenvoltura e crescimento } \\
\text { aprendidos. O conceito se relaciona à capacidade } \\
\text { de funcionar em um alto nível psicológico, dado à } \\
\text { capacidade do indivíduo e experiências anteriores }\end{array}$ & $\begin{array}{l}\text { Endireitar-se, } \\
\text { desenvolver-se, } \\
\text { crescer, aprender, } \\
\text { funcionar em alto } \\
\text { nível }\end{array}$ \\
\hline $\begin{array}{l}\text { Hamel e } \\
\text { Valikangas } \\
(2003)\end{array}$ & $\begin{array}{l}\text { Organiza- } \\
\text { cional }\end{array}$ & $\begin{array}{l}\text { Resiliência se refere à capacidade de reconstrução } \\
\text { contínua }\end{array}$ & Reconstruir \\
\hline $\begin{array}{l}\text { Horne e Orr } \\
(1998)\end{array}$ & $\begin{array}{l}\text { Organiza- } \\
\text { cional }\end{array}$ & $\begin{array}{l}\text { Resiliência é a qualidade fundamental para responder de } \\
\text { forma produtiva à alteração significativa que perturba o } \\
\text { padrão esperado de um evento sem a introdução de um } \\
\text { período prolongado de comportamento regressivo }\end{array}$ & $\begin{array}{l}\text { Responder de forma } \\
\text { produtiva }\end{array}$ \\
\hline $\begin{array}{l}\text { McDonald } \\
(2006)\end{array}$ & $\begin{array}{l}\text { Organiza- } \\
\text { cional }\end{array}$ & $\begin{array}{l}\text { Resiliência transmite as propriedades de ser capaz de } \\
\text { se adaptar aos requisitos do ambiente e de ser capaz de } \\
\text { controlar a variabilidade ambiental }\end{array}$ & $\begin{array}{l}\text { Controlar, } \\
\text { organizar, adaptar }\end{array}$ \\
\hline $\begin{array}{l}\text { Burnard } \\
\text { e Bhamra } \\
(2011)\end{array}$ & $\begin{array}{l}\text { Sistemas } \\
\text { organiza- } \\
\text { cionais }\end{array}$ & $\begin{array}{l}\text { Resiliência é a propriedade emergente de sistemas } \\
\text { organizacionais que se relaciona com as qualidades e } \\
\text { capacidades inerentes e adaptáveis que permitem uma } \\
\text { capacidade de organização adaptativa desses sistemas } \\
\text { durante períodos turbulentos, adotando uma abordagem } \\
\text { proativa à ameaça e mitigação de riscos }\end{array}$ & $\begin{array}{l}\text { Organizar, adaptar, } \\
\text { adotar abordagem } \\
\text { proativa, mitigar }\end{array}$ \\
\hline $\begin{array}{l}\text { Briguglio et } \\
\text { al. (2009) }\end{array}$ & $\begin{array}{l}\text { Sistemas } \\
\text { econômicos }\end{array}$ & $\begin{array}{l}\text { Resiliência é a capacidade induzida pela política de uma } \\
\text { economia de resistir ou de recuperar-se dos efeitos de } \\
\text { choques ocorridos }\end{array}$ & Resistir, recuperar \\
\hline $\begin{array}{l}\text { Soumagne } \\
\text { et al. (2009) }\end{array}$ & $\begin{array}{l}\text { Varejo } \\
\text { urbano }\end{array}$ & $\begin{array}{l}\text { A resiliência de um sistema de varejo urbano é definida } \\
\text { como a capacidade de diferentes tipos de varejo em se } \\
\text { adaptarem às mudanças, crises ou choques que desafiam } \\
\text { o equilíbrio do sistema sem deixar de desempenhar suas } \\
\text { funções de forma sustentável }\end{array}$ & $\begin{array}{l}\text { Adaptar, } \\
\text { desempenhar } \\
\text { funções de forma } \\
\text { sustentável }\end{array}$ \\
\hline
\end{tabular}

Quadro 1 - Definições de Resiliência

Fonte: elaboração própria. 
Como é possível visualizar no Quadro 1, o conceito é retratado, amiúde, como uma capacidade ou processo, cujas principais propriedades são: adaptar, manter, absorver, recuperar, reorganizar e resistir. Da mesma forma, embora menos reiteradas, outras importantes propriedades são: superar, aprender, crescer, desenvolver, reagir e renovar. Quanto às variáveis para as quais a resiliência versa diligência, destacam-se: vulnerabilidade, risco, mudança, variabilidade, turbulência, perturbação, adversidade, choque e estresse.

Os conceitos retratados estão consonantes, principalmente, com a segunda perspectiva observada por Lee et al. (2013), a qual aborda a resiliência como um processo dinâmico. Dessa forma, observa-se que a qualidade de ser resiliente indica a capacidade de se manter ou atingir um equilíbrio dinâmico em harmonia com a reorganização advinda da adaptação às adversidades enfrentadas (HOLLING, 1973; PATON; SMITH; VIOLANTI, 2000; WALKER et al., 2002; HAMEL; VALIKANGAS, 2003; WALKER et al., 2004; LUTHANS; VOGELGESANG; LESTER, 2006; MCDONALD, 2006; RUTTER, 2006; BRIGUGLIO et al., 2009; SOUMAGNE et al., 2009; HERRMAN et al., 2011; LEE et al., 2013). Ademais, embora a inobservância de um conceito que englobe todas as características necessárias para a sua definição em todas as ciências, as propriedades da resiliência permanecem praticamente uniformes.

\subsection{RESILIÊNCIA EM SISTEMAS DE VAREJO URBANO}

No que concerne às características da resiliência em sistemas econômicos, é importante se destacar que a análise do conceito em tais ambientes se apropria de elementos vinculados a aspectos individuais. Ponomarov e Holcomb (2009) elencam como características imprescindíveis à resiliência individual as seguintes propriedades: prontidão, preparo, resposta, adaptação, recuperação e ajuste; ao passo que Keong e Mei (2010) pontuam como quatro importantes traços de resiliência, de indivíduos em organizações, a flexibilidade, a motivação, a perseverança e o otimismo. É possível observar que tais conceitos se adequariam, com alguma variação, ao escopo econômico.

Contudo, embora comunidades e sistemas urbanos se apropriem desses traços e elementos, a interconexão entre a profusão de agentes que os compõem altera a concepção de resiliência, porquanto dá margem ao surgimento de fatores emergentes que inexistem no espectro individual (BHAMRA; DANI; BURNARD, 2011; BURNARD; BHAMRA, 2011), dado que se caracterizariam como sistemas complexos (DOOLEY, 1997; COMFORT et al. 2001; CRICHTON; RAMSAY; KELLY, 2009; ERKIP; KIZILGÜN; AKINCI, 2014).

Assim, fatores emergentes que, aparentemente, influenciam negativamente o contexto mercadológico, tais como risco, mudanças, crises econômicas, surgimento de concorrentes, instalação de competidores nas cercanias, entre outros, são, até certo ponto importantes para a solidificação da resiliência de um determinado sistema (ERKIP; KIZILGÜN; AKINCI, 2014; FRANCIS; BEKERA, 2014).

Fatores emergentes, dessa forma, cumprem um destacado papel na exposição das vulnerabilidades de um sistema, uma vez que põe à prova as suas qualidades. A vulnerabilidade, definida como o grau com o qual um sistema está propenso a experimentar dificuldade devido à exposição a ameaças e perturbações, é um conceito inerente aos sistemas econômicos (HOLLING, 2001; BHAMRA; DANI; BURNARD, 2011). Uma avaliação das próprias vulnerabilidades é capaz de indicar as diretrizes apropriadas às ações que devem ser implementadas, com o objetivo de se ampliar a resiliência sistêmica (FRANCIS; BEKERA, 2014). Tais ações podem ser direcionadas no sentido de se reforçar a resistência a eventos externos, reorganizar recursos e realizar ajustes estruturais, acomodar prováveis mudanças ou intensificar a preparação para operações de recuperação (FRANCIS; BEKERA, 2014). Dessa forma, não prescinde a tais sistemas a identificação de suas vulnerabilidades, extraindo o aprendizado 
necessário de suas fraquezas para a superação das futuras disrupções que se lhes apresentarão (HOLLING, 2001; BHAMRA; DANI; BURNARD, 2011; FRANCIS; BEKERA, 2014).

Do mesmo modo, para que se superem as adversidades incorridas no curso de atuação dos mercados, também faz-se necessário a ciência de suas próprias fortalezas. A compreensão e o desenvolvimento de suas capacidades e diferenciais competitivos possibilitam uma posição privilegiada de mercado, o que, invariavelmente, compete para a adaptação, manutenção, recuperação e crescimento desses sistemas; ações indissoluvelmente associadas ao conceito de resiliência (HOLLING, 2001; BHAMRA; DANI; BURNARD, 2011; FRANCIS; BEKERA, 2014).

A importância do estudo da resiliência em sistemas de varejo urbano reside, portanto, no fato de que tal análise, além de expor as fortalezas, é capaz de identificar possíveis vulnerabilidades, apontar prováveis riscos vinculados ao sistema e auxiliar na implementação de ações importantes para a continuidade e desenvolvimento desses mercados (FRANCIS; BEKERA, 2014).

Ao passo que na psicologia as ações para a cristalização da resiliência em âmbito individual são voltadas para a ampliação da esfera social, intensificação dos laços familiares, autoconhecimento, entre outras, a literatura referente à resiliência elenca medidas importantes de incremento dessa propriedade em sistemas com características culturais diferentes (MEDD; MARVIN, 2005; SOUMAGNE et al., 2009; OZUDURU; VAROL; ERCOSKUN, 2014; PETRESCU; BHATLI, 2013; ERKIP; KIZILGÜN; AKINCI, 2014). No Quadro 2, a seguir, são apontadas as forças de resiliência erigidas a do arquétipo teórico relacionado aos sistemas de varejo urbano. Dessa forma, três subsistemas foram destacados, quais sejam: político, relacionado à esfera governamental; econômico, referente ao sistema de varejo em si, i.e., ao conjunto de comerciantes; e de inovação, relacionado especificamente aos comerciantes, unidades constituintes elementares.

\begin{tabular}{|c|c|c|c|}
\hline \multicolumn{4}{|c|}{ Esfera Política } \\
\hline i. & Imagem positiva do ambiente & ii. & Instalações apropriadas \\
\hline iii. & Acessibilidade & iv. & Defesa contra concorrentes \\
\hline v. & Segurança & vi. & Educação financeira e empresarial \\
\hline vii. & Higiene & viii. & Turismo \\
\hline \multicolumn{4}{|c|}{ Esfera Econômica } \\
\hline i. & Crescimento econômico & ii. & Liderança comprometida \\
\hline iii. & Oferta de atrações de cultura e lazer & iv. & Festivais \\
\hline V. & Planejamento & vi. & Estética/Plástica \\
\hline \multicolumn{4}{|c|}{ Esfera de Inovação } \\
\hline i. & Aprendizagem & ii. & Estratégias de promoção \\
\hline iii. & Competitividade & iv. & Auto-organização \\
\hline v. & Relações amistosas & vi. & Inovação \\
\hline
\end{tabular}

Quadro 2 - Forças de resiliência de sistemas de varejo urbano

Fonte: elaboração própria.

Embora sistemas varejistas urbanos sejam caracterizados como sistemas econômicos, é importante que se distingam os dois conceitos. Sistema econômico é uma expressão que se refere a qualquer arranjo produ- tivo ou de distribuição de bens ou serviços, assim como a alocação de recursos em uma sociedade, que tenha como escopo o superávit financeiro. Exemplos como clusters, setores da economia, a economia de um país 
e sistemas de varejo urbano se enquadram nessa definição.

Sistemas de varejo urbano, por seu turno, são ambientes que comportam vários negócios distintos que compartilham uma mesma infraestrutura e imagem; o que ocasiona, dessa forma, interdependência entre seus agentes (MEDD; MARVIN, 2005; SOUMAGNE et al., 2009; KÄRRHOLM; NYLUND; DE LA FUENTE, 2014; ERKIP; KIZILGÜN; AKINCI, 2014). O termo urbano inserido à expressão tem o intuito de restringir a ideia de sistemas de varejo ao espaço físico em que se situam, em detrimento de uma percepção em termos macroeconômicos, como setores e segmentos de varejo de uma nação.

O conceito de equilíbrio para sistemas econômicos não é entendido de maneira estática, dado que permanecer estagnado em um mesmo patamar, qualquer que seja o indicador em análise, se taxa de crescimento, estrutura econômica, índice de emprego, acesso a financiamento para investimento, competitividade, inovação, cultura empreendedora, dentre outros, não se traduz em desenvolvimento (SIMMIE; MARTIN, 2010; MARTIN, 2012). Em vez de retornarem a um estado anterior de equilíbrio, as regiões, setores e países estão se reconfigurando e se adaptando constantemente ao ambiente econômico global.

Resiliência econômica, dessa forma, é entendida não como uma habilidade ou capacidade de um sistema, mas como um processo inelutavelmente presente no contexto dos sistemas econômicos, representando o processo de superação de choques, em detrimento do equilíbrio paralisante (BRIGUGLIO, 2016; BASTAMINIA; REZAEI; DASTOORPOOR, 2017). O processo de resiliência de um sistema econômico pode ter como parâmetro um ou a combinação de alguns dos indicadores supracitados (SIMMIE; MARTIN, 2010; MARTIN, 2012). Tal dinâmica é evidenciada no Gráfico 1, a seguir.

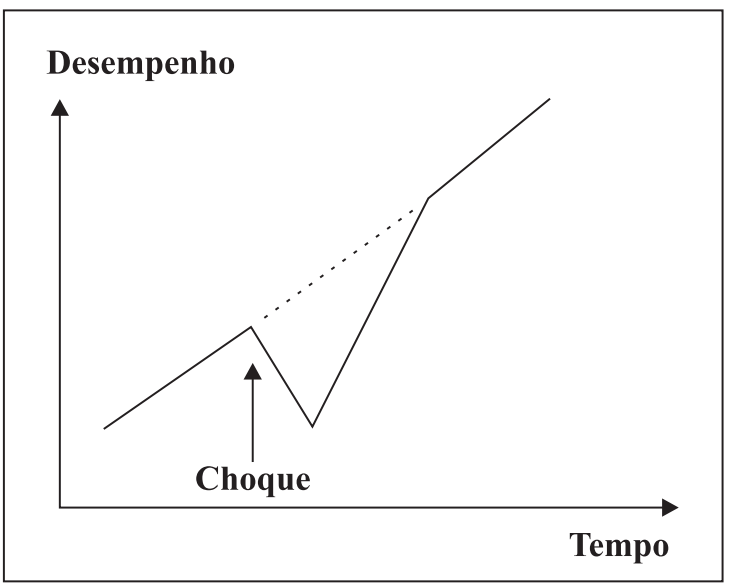

Gráfico 1 - Processo de resiliência - retorno ao equilíbrio dinâmico inicial

Fonte: adaptado de Martin (2012).

Como é possível observar, o sistema voltou a apresentar, após o período de abalo, o mesmo desempenho que demonstrará anteriormente, dado que passou a exibir coeficiente angular idêntico. Esse período de queda e recuperação do desenvolvimento, até atingir os índices novamente positivos, é conceituado por Martin (2012) como histerese; conceito resgatado das ciências naturais que significa atraso ou retardamento do efeito sobre um objeto devido à variação, aumento ou diminuição, das forças externas que atuam sobre ele.

Embora o processo de resiliência que atua sobre sistemas econômicos os impele ao crescimento em algum ponto futuro, nem sempre esse crescimento consegue acompanhar a taxa de desenvolvimento inicial. A variação do coeficiente angular do desempenho apresentado pelo sistema, após a retomada do crescimento, pode ser nula ou negativa, como é possível observar no Gráfico 2, a seguir. 


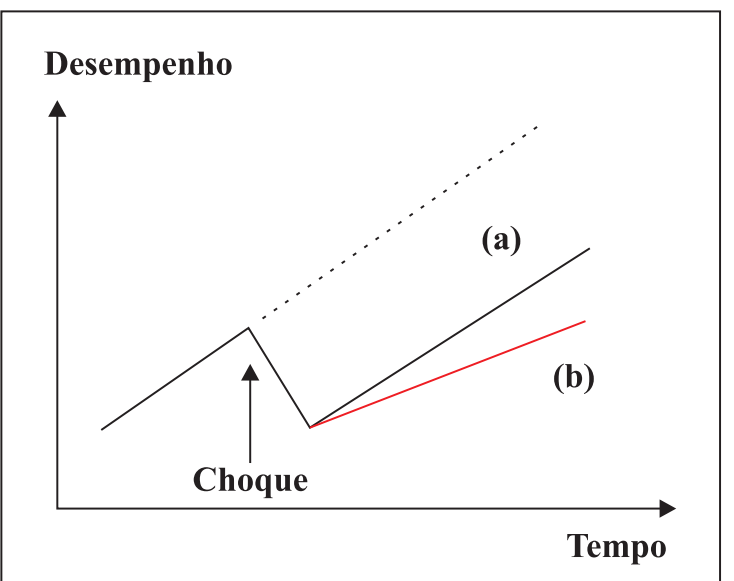

Gráfico 2 - Processo de resiliência - equilíbrios dinâmicos inferiores ao inicial

Fonte: adaptado de Martin (2012).

Quando o equilíbrio dinâmico é inferior ao inicial, duas situações podem ocorrer ao se alcançar novamente o desenvolvimento. Na situação (a), a variação do coeficiente angular é nula e a reta é, simplesmente, deslocada para baixo. Tal circunstância indica que o índice de desenvolvimento continuará o mesmo, embora a cifras menores. Por outro lado, ao se obter uma variação negativa do coeficiente angular, demonstrada pela situação (b), observa-se que o sistema voltou a apresentar crescimento, contudo, com uma menor variação percentual.

A retomada do crescimento pelo sistema, no entanto, pode alcançar valores que superem a taxa inicial apresentada antes do choque. Em tal contexto, o equilíbrio dinâmico exprimido pelo sistema é superior ao preliminar, porquanto revele, após ultrapassar a taxa inicial, uma variação do coeficiente angular nula ou positiva. Essa relação pode ser visualizada no Gráfico 3, a seguir.

De acordo com o gráfico, ao se ultrapassar a taxa inicial de crescimento, o sistema pode apresentar uma entre duas situações. Caso o sistema atinja uma variação do coeficiente angular positiva, como evidenciado pela situação (a), a reta é deslocada para cima, indicando a estabilidade da taxa de crescimento; no entanto, com valores superiores. Contudo, se o sistema continuar com a variação positiva de desempenho após a retomada do crescimento, revelado pelo contexto (b), obter-se-á uma situação de desenvolvimento superior ao nível inicial.
Contudo, quanto à resiliência de mercados e outros sistemas econômicos, uma ressalva deve ser feita, haja vista que a economia das nações, por exemplo, embora enfrentem crises periódicas, simplesmente não declaram bancarrota e desaparecem; ao contrário do que pode ocorrer com os sistemas urbanos. Com efeito, crescer é um movimento não somente necessário, mas obrigatório a sistemas econômicos nacionais. Dessa forma, manter-se estável, ao se selecionar um ou alguns dos indicadores econômicos como parâmetro, pode indicar, para os mercados, resiliência frente à ruína e à insolvência.

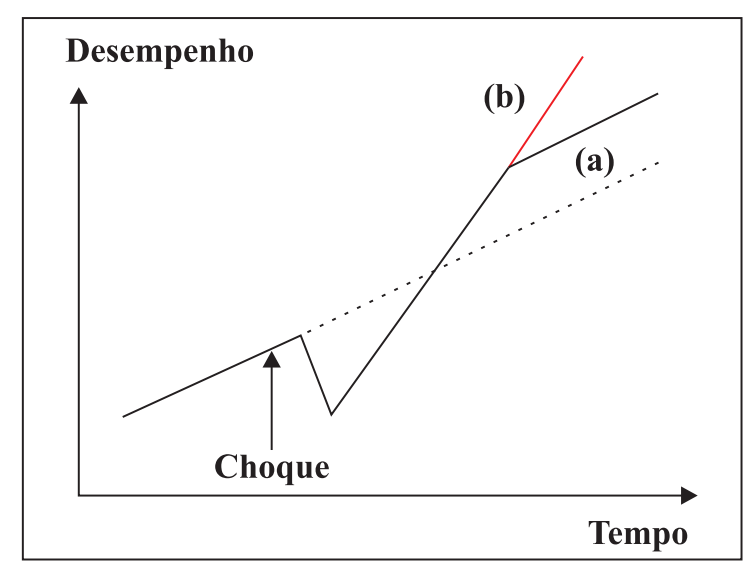

Gráfico 3 - Processo de resiliência - equilíbrios dinâmicos superiores ao inicial

Fonte: adaptado de Martin (2012).

Um modelo gráfico com as proposições apresentadas, ou seja, com um sistema apresentando desempenho constante após a influência de algum estressor externo, pode ser visualizado no Gráfico 4, a seguir.

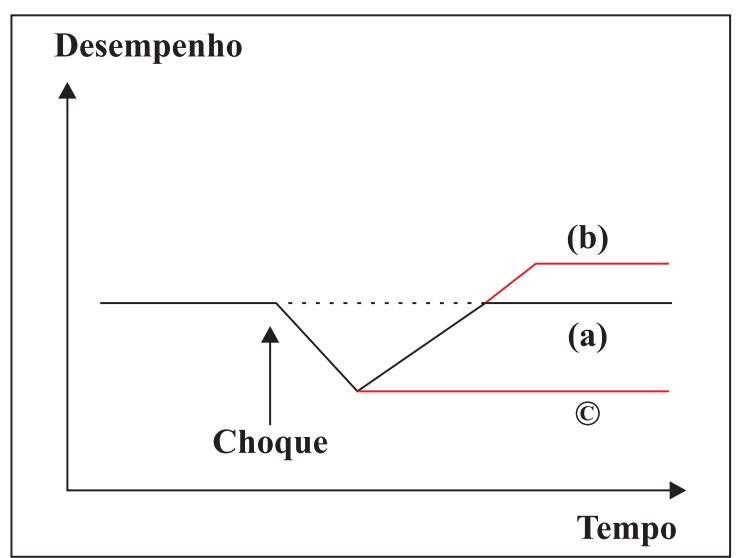

Gráfico 4 - Possibilidade de equilíbrio no processo de resiliência em sistemas de varejo Fonte: elaboração própria. 
Na situação (a), não há variação dos coeficientes angular e linear, levando o sistema a permanecer no mesmo patamar registrado antes do choque. Por outro lado, uma vez apresentada uma variação negativa do coeficiente linear, demonstrada pela situação (c), observa-se que a reta é deslocada para baixo, levando o sistema a permanecer em equilíbrio, porém a valores menores. Por fim, a situação (b) demonstra uma variação positiva no coeficiente linear, ocasionando o deslocamento vertical da reta a um nível superior ao registrado no período inicial.

É importante se ressaltar que, embora seja possível ter como parâmetro indicadores de ordem puramente econômica, tais como taxa de crescimento, infraestrutura, índice de emprego, acesso a financiamento, entre outros, há fatores não diretamente vinculados a aspectos financeiros que favorecem o processo de resiliência do sistema. Dessa forma, de acordo com as acepções levantadas, o equilíbrio dinâmico não adviria do simples crescimento pecuniário ou do acréscimo de eficiência de qualquer outro parâmetro em análise, uma vez que a reta de desenvolvimento do sistema de varejo pode permanecer constante.

Desse modo, um sistema de varejo pode apresentar volume de venda ou receita total constante ao longo de vários períodos. No entanto, há sempre fatores implícitos sendo incrementados durante esses longos períodos, como a aprendizagem, potencial inovativo, coesão entre os agentes, competitividade, entre outros (SIMMIE; MARTIN, 2010; MARTIN, 2012).

Com base nas acepções teóricas emersas ao longo do estudo, é possível sintetizar um modelo de desenvolvimento dos sistemas de varejo urbano; apresentado no Figura 1, a seguir.

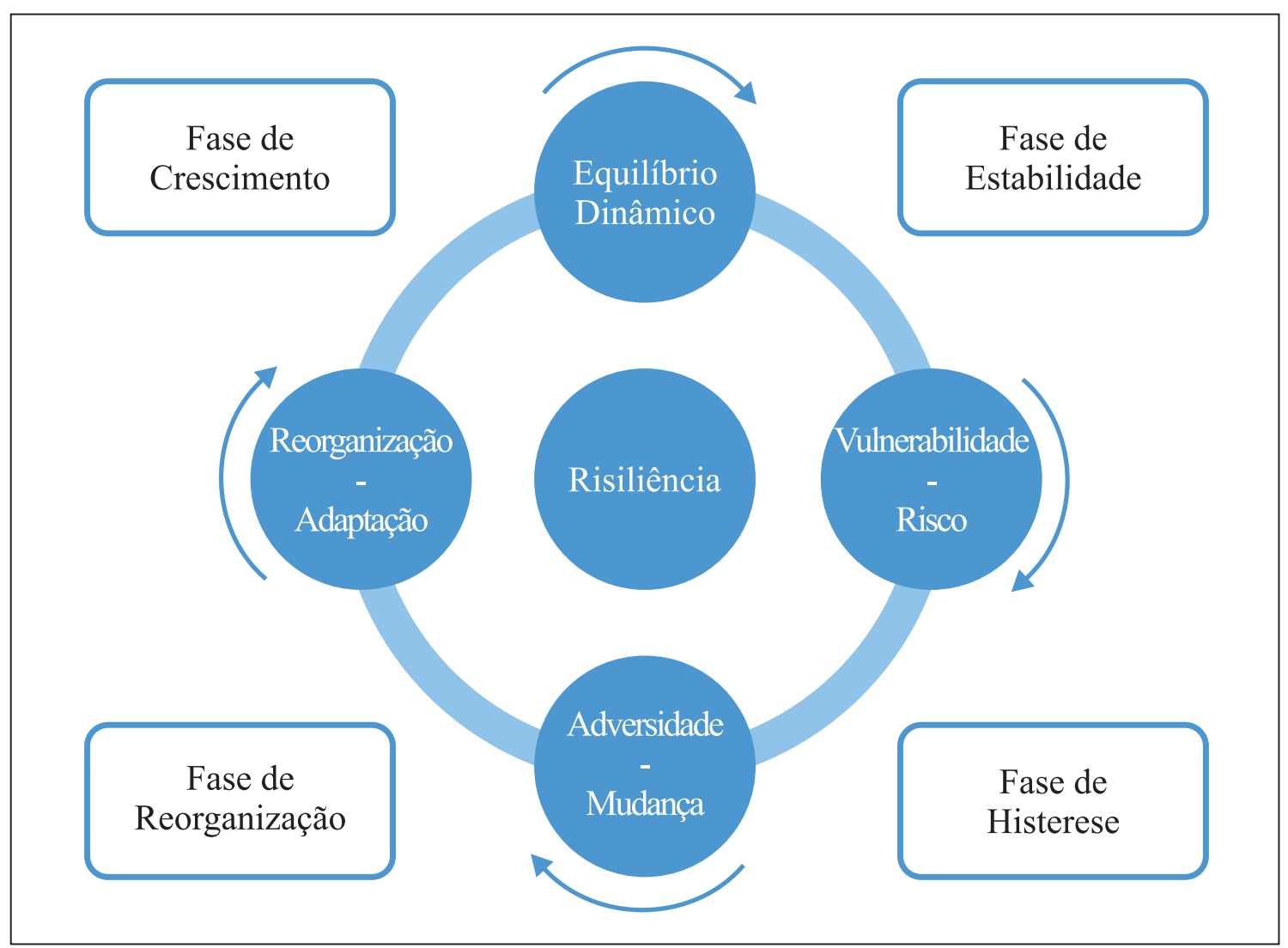

Figura 1 - Modelo de desenvolvimento dos sistemas de varejo urbano

Fonte: elaboração própria. 
A resiliência é a engrenagem central que faz girar todo o processo de desenvolvimento do sistema de varejo urbano. Como evidencia a Figura 1, quatro conceitos basilares, resultantes da confluência de fatores tanto internos quanto externos, competem para a formação da resiliência; quais sejam: equilíbrio dinâmico, vulnerabilidade/risco, adversidade/mudança e reorganização/adaptação.

Do mesmo modo, como revela o modelo, a disposição de tais fatores no processo de evolução cíclica do sistema acarreta o surgimento de quatro fases de desenvolvimento. A fase de estabilidade, decorrente do alcance do equilíbrio dinâmico e sustentável, representa o começo no qual o sistema acumula recursos e potencial competitivo para o enfrentamento de suas vulnerabilidades e riscos externos. Este estágio precede a fase de histerese ou destruição criativa, na qual o sistema desconstrói elementos estruturais obsoletos e limitantes, a fim de superar as adversidades advindas do ambiente. Como consequência, o sistema adentra em um movimento em direção à reorganização, fase na qual passa a se adaptar às alterações provocadas pelas mudanças nos estímulos externos. Por fim, inicia-se o período de crescimento, até que se atinja novamente o equilíbrio dinâmico.

\section{CONSIDERAÇÕES FINAIS}

Com o objetivo de investigar as principais propriedades e características relacionadas à resiliência em sistemas de varejo urbano é possível afirmar que este estudo obteve êxito; dado que, além de se erigir uma definição de resiliência voltada a tais ambientes, foram analisadas as principais propriedades relacionadas ao conceito em sistemas econômicos. Ademais, foram comparadas criticamente as diferenças teóricas existentes entre os processos de desenvolvimento dos sistemas econômicos e sistemas de varejo urbano.

Os sistemas de varejo urbano, assim como os demais sistemas econômicos, apresentam processos de resiliência que os impelem a patamares cada vez maiores de equilíbrio. Ressalta- -se, contudo, que há uma peculiaridade nos sistemas urbanos que os diferenciam dos sistemas econômicos: a possibilidade de falir. Manter-se estável, dessa forma, faz parte do seu processo de resiliência. São comenos importantes para o acúmulo de potencial competitivo, uma vez que a estabilidade pressupõe uma manutenção dinâmica de seus elementos e propriedades.

O equilíbrio estático, por outro lado, traduz-se na morte do sistema; porquanto signifique a extinção do processo cíclico de retenção de recursos competitivos, destruição criativa, reorganização estrutural e crescimento. Resiliência, dessa forma, seria a capacidade de se manter ou atingir um equilíbrio dinâmico em harmonia com a reorganização advinda da adaptação às adversidades enfrentadas.

É importante se acentuar que a presença de fatores emergentes, que aparentemente influenciariam negativamente o ambiente econômico do sistema, são, em certa medida, imprescindíveis para a cristalização de sua resiliência. Destarte, o enfrentamento e a superação tanto das vulnerabilidades presentes no sistema quanto das adversidades provenientes do ambiente externo são importantes propulsores da resiliência.

Como sugestões para pesquisas futuras, aponta-se a criação de indicadores operacionais de resiliência; tema que tem recebido pouca atenção da literatura. Tais indicadores teriam como intuito analisar, por meio de parâmetros objetivos e sistemáticos, o processo de evolução dos sistemas de varejo urbano, sinalizando as fases de desenvolvimento pela qual o sistema atravessa e quais propriedades e elementos deve relevar prioritariamente no momento.

Do mesmo modo, sugere-se a análise dos sistemas de varejo urbano por meio da abordagem da Teoria Geral dos Sistemas. A perspectiva possibilita, por meio de uma lente teórica adequada ao estudo de tais ambientes, uma visão holística dos seus elementos constituintes e suas interconexões, dos fluxos de troca de materiais entre o sistema e o meio, além de permitir a análise do seu processo de desenvolvimento. 


\section{RESILIENCE IN URBAN RETAIL SYSTEMS}

\begin{abstract}
This essay aims to investigate the main properties and characteristics related to resilience in urban retail systems. For that, by means of the analysis of the theoretical aspects concerned with resilience present in studies of several disciplines, we seek to build a concept of resilience specifically to urban retail systems and to critically analyze the extent of theoretical differences between the process of development of economic and the urban retail systems. Based on analysis carried out, we highlight that the concept of resilience in those environments represents the capacity of maintaining or reaching a dynamic balance in harmony with reorganization deriving from the adaptation to faced adversity. Resilience would be a central gear that rotates the whole process of diachronic development in urban retail systems. Thus, we conclude that the struggle and the overcoming both of the vulnerabilities present in the system and the adversities derived from the external environment are an important propellant for the resilience.
\end{abstract}

Keywords: Resilience. Urban retail systems. Dynamic balance. Development.

\section{RESILIENCIA EN SISTEMAS DEL SECTOR MINORISTA URBANO}

\section{RESUMEN}

Este ensayo tiene por objetivo general investigar las principales propiedades y características relacionadas a la resiliencia en sistemas del sector minorista urbano. Para tal, por intermedio del análisis de aspectos teóricos relativos a la resiliencia en estudios de diversas asignaturas, se busca construir un concepto de resiliencia específico de sistemas del sector minorista urbano y analizar críticamente las distinciones teóricas existentes entre los proce- sos de desarrollo de los sistemas económicos y de los sistemas del sector minorista urbano. Con base en los análisis realizados, se observa que, para tales ambientes, resiliencia representa la capacidad de mantenerse o lograr un equilibrio dinámico en harmonía con la reorganización proveniente de la adaptación a las adversidades enfrentadas. La resiliencia sería el engranaje central que hace girar todo el proceso de desarrollo diacrónico de los sistemas del sector minorista urbano. Así, se concluí que el enfrentamiento y la superación tanto de las vulnerabilidades presentes en el sistema cuanto de las adversidades provenientes del ambiente externo son importantes propulsores de la resiliencia.

Palabras-clave: Resiliencia. Minoristas urbanos. Equilibrio dinámico. Desarrollo.

\section{REFERÊNCIAS}

BACCHI, G. A.; PINHEIRO, D. R. Entre o Tripalium e a Resiliência: um estudo sobre a correlação entre o assédio moral no trabalho e a resiliência. In: ENCONTRO DE GESTÃO DE PESSOAS E RELAÇÕES DE TRABALHO, 3., 2011, João Pessoa. Anais... João Pessoa: Anpad, 2011.

BASTAMINIA, A.; REZAEI, M. R.; DASTOORPOOR, M. Identification and evaluation of the components and factors affecting social and economic resilience in city of Rudbar, Iran. International Journal of Disaster Risk Reduction, v. 22, p. 269-280, 2017.

BHAMRA, R.; DANI, S.; BURNARD, K. Resilience: the concept, a literature review and future directions. International Journal of Production Research, v. 49, n. 18, p. 5375-5393, 2011.

BRANDÃO, J. M.; MAHFOUD, M.; GIANORDOLI-NASCIMENTO, I. F. A construção do conceito de resiliência em psicologia: discutindo as origens. Paidéia, v. 21, n. 49, p. 263-271, 2011. 
BRIGUGLIO, L. et al. Economic vulnerability and resilience: concepts and measurements. Oxford development studies, v. 37, n. 3, p. 229-247, 2009.

BRIGUGLIO, L. P. Exposure to external shocks and economic resilience of countries: evidence from global indicators. Journal of Economic Studies, v. 43 n. 6, p. 1057-107, 2016.

BUENO, N. P. Assessing the resilience of small socio-ecological systems based on the dominant polarity of their feedback structure. System Dynamics Review, v. 28, n. 4, p. 351-360, 2012.

BURNARD, K.; BHAMRA, R. Organisational resilience: development of a conceptual framework for organisational responses. International Journal of Production Research, v. 49, n. 18, p. 5581-5599, 2011.

CANGUSSU, E. T.; SACHUK, M. I. Compreendendo a resiliência em ambientes de trabalho: experiências vividas por empresários egressos do Banco do Brasil. Qualitas Revista Eletrônica, v. 15, n. 2, p. 2014.

CARPENTER, S. et al. From metaphor to measurement: resilience of what to what? Ecosystems, v. 4, n. 8, p. 765-781, 2001.

CARVALHO, V. D. de. et al. Resiliência e socialização organizacional entre servidores públicos brasileiros e noruegueses. RAC-Revista de Administração Contemporânea, v. 15, n. 5, p. 815-833, 2011.

COMFORT, L. K. et al. Complex systems in crisis: Anticipation and resilience in dynamic environments. Journal of contingencies and crisis management, v. 9, n. 3, p. 144-158, 2001.

CRICHTON, M. T.; RAMSAY, C. G.; KELLY, $\mathrm{T}$. Enhancing organizational resilience through emergency planning: learnings from cross sec- toral lessons. Journal of Contingencies and Crisis Management, v. 17, n. 1, p. 24-37, 2009.

DOOLEY, K. J. A complex adaptive systems model of organization change. Nonlinear dynamics, psychology, and life sciences, v. 1, n. 1, p. 69-97, 1997.

ERKIP, F.; KIZILGÜN, Ö.; AKINCI, G. M. Retailers' resilience strategies and their impacts on urban spaces in Turkey. Cities, v. 36, p. 112$120,2014$.

FRANCIS, R.; BEKERA, B. A metric and frameworks for resilience analysis of engineered and infrastructure systems. Reliability Engineering \& System Safety, v. 121, p. 90103, 2014.

HAMEL, G.; VALIKANGAS, L. The quest for resilience. Harvard business review, v. 81, n. 9, p. 52-65, 2003.

HERRMAN, H. et al. What is resilience? The Canadian Journal of Psychiatry, v. 56, n. 5, p. 258-265, 2011.

HOLLING, C. S. Resilience and stability of ecological systems. Annual review of ecology and systematics, v. 4, n. 1, p. 1-23, 1973.

. Understanding the complexity of economic, ecological, and social systems. Ecosystems, v. 4, n. 5, p. 390-405, 2001.

HOLLNAGEL, E.; WOODS, D. D.; LEVESON, N. Resilience engineering: concepts and precepts. Surrey: Ashgate Publishing, Ltd., 2006.

HORNE, J. F.; ORR, J. E. Assessing behaviours that create resilient organisations. Employment Relations Today, v. 24, n. 4, p. 29-39, 1998.

KÄRRHOLM, M.; NYLUND, K.; DE LA FUENTE, P. P. Spatial resilience and urban 
planning: addressing the interdependence of urban retail areas. Cities, v. 36, p. 121-130, 2014.

KEONG, F. W. F.; MEI, L. Y. Sustainable development: the effect of adopting green technology on small and medium enterprises'(smes) business resilience and competitiveness. In: INTERNATIONAL CONFERENCE ON BUSINESS AND ECONOMIC RESEARCH (ICBER 2010), 2010, Malaysia. Proceedings... Malaysia: ICBER, 2010. p. 1516.

LEE, J. H. et al. Resilience: a meta analytic approach. Journal of Counseling \& Development, v. 91, n. 3, p. 269-279, 2013.

LUTHANS, F.; VOGELGESANG, G. R.; LESTER, P. B. Developing the psychological capital of resiliency. Human Resource Development Review, v. 5, n. 1, p. 25-44, 2006.

MARTIN, R. Regional economic resilience, hysteresis and recessionary shocks. Journal of Economic Geography, v. 12, n. 1, p. 1-32, 2012.

MCDONALD, N. Organisational resilience and industrial risk. In: HOLLNAGEL, E.; WOODS, D. D.; LEVESON, N. Resilience engineering: concepts and precepts. Surrey: Ashgate, 2006. p. 155-180.

MEDD, W.; MARVIN, S. From the politics of urgency to the governance of preparedness: A research agenda on urban vulnerability. Journal of Contingencies and Crisis Management, v. 13, n. 2, p. 44-49, 2005.

OZUDURU, B. H.; VAROL, C.; ERCOSKUN, O. Y. Dos shopping centers abate the resilience of shopping streets? The co-existence of both shopping venues in Ankara, Turkey. Cities, v. 36, p. 145-157, 2014.

PATON, D.; SMITH, L.; VIOLANTI, J. Di- saster response: risk, vulnerability and resilience. Disaster Prevention and Management: an International Journal, v. 9, n. 3, p. 173-180, 2000.

PETRESCU, M.; BHATLI, D. Consumer behavior in flea markets and marketing to the Bottom of the Pyramid. Journal of Management Research, v. 13, n. 1, p. 55, 2013.

PONOMAROV, S. Y.; HOLCOMB, M. C. Understanding the concept of supply chain resilience. The international journal of logistics management, v. 20, n. 1, p. 124-143, 2009.

POWLEY, E. H. Reclaiming resilience and safety: Resilience activation in the critical period of crisis. Human Relations, v. 62, n. 9, p. 1289-1326, 2009.

RUTTER, M. Implications of resilience concepts for scientific understanding. Annals of the New York Academy of Sciences, v. 1094, n. 1, p. 1-12, 2006.

SABBAG, P. Y. et al. Validação de escala para mensurar resiliência por meio da Teoria de Resposta ao Item (TRI). In: ENCONTRO DA ANPAD, 34., 2010, Rio de Janeiro. Anais... Rio de Janeiro: Anpad, 2010. p. 1-15.

SCHERER, L. A.; MINELLO, I. F. Resiliência e expatriação: das adversidades à dinâmica do comportamento resiliente de expatriados. Revista Alcance (Online), v. 24, n. 3, p. 329-349, 2017.

SIMMIE, J.; MARTIN, R. The economic resilience of regions: towards an evolutionary approach. Cambridge journal of regions, economy and society, v. 3, n. 1, p. 27-43, 2010.

SMITH, B. W. et al. The brief resilience scale: assessing the ability to bounce back. International journal of behavioral medicine, v. 15, n. 3, p. 194-200, 2008. 
SOUMAGNE, J. et al. Retail planning for cities sustainability (Replacis). Portugal: [s.n.], 2009.

STUMPP, E. M. New in town? On resilience and "Resilient Cities". Cities, v. 32, p. 164166, 2013.

TIMOSHENKO, Stephen P. History of strenght of materials: with a brief account of the history of theory of elasticity and theory of structures. [S.1.:s.n.], 1983.

WALKER, B. et al. Resilience management in social-ecological systems: a working hypothesis for a participatory approach. Conservation ecology, v. 6, n. 1, 2002.

WALKER, B. et al. Resilience, adaptability and transformability in social-ecological systems. Ecology and society, v. 9, n. 2, 2004.

YUNES, M. A. M. Psicologia positiva e resiliência: o foco no indivíduo e na família. Psicologia em Estudo, v. 8, p. 75-84, 2003. 